\title{
WEAK CONVERGENCE OF ASYMPTOTICALLY HOMOGENEOUS FUNCTIONS OF MEASURES
}

\author{
Françoise Demengel \\ Laboratoire d'Analyse Numérique, Université Paris-Sud, 91405 Orsay, France \\ and \\ JEFFREY RAUCH* \\ University of Michigan, Ann Arbor, Michigan 48109, U.S.A.
}

(Received 28 April 1989; received for publication 15 September 1989)

Key words and phrases: Functions of measures, weak convergence, measures.

\section{INTRODUCTION}

Functions of measures have already been introduced and studied by Goffman and Serrin [8], Reschetnyak [10] and Demengel and Temam [5]: especially for the convex case, some lower continuity results for the weak tolopology (see [5]) permit us to clarify and solve, from a mathematical viewpoint, some mechanical problems, namely in the theory of elastic plastic materials.

More recently, in order to study weak convergence of solutions of semilinear hyperbolic systems, which arise from mechanical fluids, we have been led to answer the following question: on what conditions on a sequence $\mu_{n}$ of bounded measures have we $f\left(\mu_{n}\right) \rightarrow f(\mu)$ where $\mu$ is a bounded measure and $f$ is any "function of a measure" (not necessarily convex)? We answer this question in Section 1 when the functions $f$ are homogeneous, and in Section 2 for sublinear functions; the general case of asymptotically homogeneous function is then a direct consequence of the two previous cases! An extension to $x$ dependent functions is described in Section 4. In the one dimensional case, we give in proposition 3.3 a criteria which is very useful in practice, namely for the weak continuity of solutions measures of hyperbolic semilinear systems with respect to weakly convergent Cauchy data.

Another application concerns a work in preparation [6] on measure-valued solutions for hyperbolic scalar equations.

The results of this paper were announced in [2].

\section{1. $F(\mu)$ WITH $F$ HOMOGENEOUS}

For compact Hausdorff space $\Gamma, C\left(\Gamma, \mathbb{R}^{d}\right)$ denotes the Banach space of continuous $\mathbb{R}^{d}$ valued functions normed by

$$
\|\varphi\|_{L^{\infty}}=\sup |\varphi|=\sup _{x \in \Gamma}\left(\Sigma \varphi_{i}(x)^{2}\right)^{1 / 2} .
$$

We assume throughout that $\Gamma$ is nonempty.

\footnotetext{
* Research partially supported by grant NSF DMS 86-01/83 from the United States National Science Foundation.
} 
The $\mathbb{R}^{d}$ valued Borel measures $\mu \in M\left(\Gamma, \mathbb{R}^{d}\right)$ represent the dual of $C\left(\Gamma, \mathbb{R}^{d}\right)$ with the pairing

The norm of $\mu$ is then

$$
(\mu, \varphi)=\int \varphi_{i} \mathrm{~d} \mu^{i}, \quad \mu=\left(\mu^{1}, \ldots, \mu^{d}\right) .
$$

$$
\|\mu\|=\sup \left\{\langle\mu, \varphi\rangle:\|\varphi\|_{L^{\infty}} \leq 1\right\} .
$$

The variation $|\mu| \in M(\Gamma, \mathbb{R})$ is defined by giving it values on open sets $\omega \subset \Gamma$

$$
|\mu|(\omega)=\sup \left\{\langle\mu, \varphi\rangle: \varphi \in C\left(\Gamma, \mathbb{R}^{d}\right),\|\varphi\|_{L^{\infty}} \leq 1, \operatorname{supp} \varphi \subset \omega\right\} .
$$

Then $\|\mu\|=|\mu|(\Gamma)$ is the total variation. With these definitions, if $\mu=g v, v \in M(\Gamma, \mathbb{R})$ then

$$
\|\mu\|=\int\left(\Sigma g_{i}(x)^{2}\right)^{1 / 2} \mathrm{~d}|v| .
$$

Norm convergence of a sequence of measures is denoted with an arrow $\rightarrow$, and weak star convergence is denoted with a half arrow $\rightarrow$. Thus if $\mu_{k} \in M\left(\Gamma, \mathbb{R}^{d}\right), k=1,2, \ldots$ and $\mu \in M\left(\Gamma, \mathbb{R}^{d}\right)$,

$$
\begin{array}{ll}
\mu_{k} \rightarrow \mu & \text { iff }\left\|\mu_{k}-\mu\right\| \rightarrow 0 \\
\mu_{k} \rightarrow \mu & \text { iff } \forall \varphi \in C\left(\Gamma, \mathbb{R}^{d}\right),\left\langle\mu_{k}, \varphi\right) \rightarrow\langle\mu, \varphi\rangle .
\end{array}
$$

If $F: \mathbb{R}^{d} \rightarrow \mathbb{R}$ is positively homogeneous of degree 1 , and Borel measurable, then for $\mu \in M\left(\Gamma, \mathbb{R}^{d}\right), F(\mu) \in M(\Gamma, \mathbb{R})$ is defined as follows (see [1, Section 5, pp. 65-67]). Choose a nonnegative $v \in M(\Gamma, \mathbb{R})$ so that each component $\mu^{i}$ is absolutely continuous with respect to $v$. Then $\mu=g \nu$ with $g: \Gamma \rightarrow \mathbb{R}^{d}$ Borel measurable and integrable $\mathrm{d} v$ and $F(\mu)$ is defined by $F(\mu) \equiv F(g) v$. That this is independent of the choice of $v$ is proved as follows. For two choices $v_{1}$ and $v_{2}$, choose a third $v_{3}$, with $v_{i} \ll v_{3}, i=1,2$ then express all three representations as multiples of $v_{3}$.

Immediate consequences of the definition are:

(1.2) If $\mu_{j} \in M\left(\Gamma, \mathbb{R}^{d}\right) j=1,2$ are mutually singular, $\mu_{1} \perp \mu_{2}$, then $F\left(\mu_{1}+\mu_{2}\right)=F\left(\mu_{1}\right)+$ $F\left(\mu_{2}\right)$.

(1.3) If $g: \Gamma \rightarrow \mathbb{R}$ is a nonnegative Borel measureable function integrable $\mathrm{d} \mu$ then $F(g \mu)=g F(\mu)$.

Example 1.4. If $F(\xi)=\left(\xi^{i}\right)_{+}$then $F(\mu)$ is the positive part of $\mu^{i}$.

Example 1.5. If $F(\xi) \equiv|\xi| \equiv\left(\Sigma\left(\xi^{i}\right)^{2}\right)^{1 / 2}$ then $F(\mu) \equiv|\mu|$ is the variation of $\mu$. If $\Omega \subset \mathbb{R}^{n}$ is open and $u: \Omega \rightarrow \mathbb{R}$ is of bounded variation on $\Omega$ then $F\left(\mathrm{~d} x, \partial u / \partial x_{1}, \ldots, \partial u / \partial x_{n}\right)$ is the surface area measure for the graph of $u$ (see [8]).

Example 1.6. If $\Omega \subset \mathbb{R}^{n}$ is open $u: \Omega \rightarrow \mathbb{R}$ is convex then $\left[\operatorname{det}\left[\partial^{2} u / \partial x_{i} \partial x_{j}\right]\right)^{1 / n}$ is the MongeAmpère measure of the graph, related to the Gauss curvature (see [9]).

The next theorem gives basic results concerning the continuity of the map $\mu, F \rightarrow F(\mu)$ with the norm topology on $M\left(\Gamma, \mathbb{R}^{d}\right)$. 
Theorem 1.7. If $F$ and $G$ are two Borel functions homogeneous of degree 1 and $|F(\xi)-G(\xi)| \leq \delta$ for all $|\xi|=1$ then

$$
|F(\mu)-G(\mu)| \leq \delta|\mu| .
$$

If $F$ is Lipschitz continuous and $\Lambda$ is a Lipshitz constant $|F(\xi)-F(\eta)| \leq \Lambda|\xi-\eta|$ for all $\xi, \eta \in \mathbb{R}^{d}$, then for $\mu_{j} \in M\left(\Gamma, \mathbb{R}^{d}\right) j=1,2$

$$
\left|F\left(\mu_{1}\right)-F\left(\mu_{2}\right)\right| \leq \Lambda\left|\mu_{1}-\mu_{2}\right| \text {. }
$$

For $F$ positively homogeneous and Borel, the map $\mu \rightarrow F(\mu)$ is continuous for the norm topology in $M\left(\Gamma, \mathbb{R}^{d}\right)$ if and only if $F \in C\left(\mathbb{R}^{d}, \mathbb{R}\right)$. In this case, the map $\mu \rightarrow F(\mu)$ is uniformly continuous on norm bounded subsets of $M\left(\Gamma, \mathbb{R}^{d}\right)$.

Proof. The first assertion is immediate. For the second choose $v \in M(\Gamma, \mathbb{R})$ with $\mu_{j} \ll v$ $j=1,2$. Then $\mu_{j}=h_{j} v$ and

$$
\left|F\left(\mu_{1}\right)-F\left(\mu_{2}\right)\right|=\left|F\left(h_{1}\right)-F\left(h_{2}\right)\right| \mathrm{d}|v| \leq \Lambda\left|h_{1}-h_{2}\right| \mathrm{d} v=\Lambda\left|\mu_{1}-\mu_{2}\right| .
$$

To prove the necessity of continuity in the third part suppose that $\xi_{k} \rightarrow \xi$ in $\mathbb{R}^{d}$. Then for any $\gamma \in \Gamma, \mu_{k} \equiv \xi_{k} \delta_{\gamma} \rightarrow \xi \delta_{\gamma}$. If $\mu \rightarrow F(\mu)$ is continuous for norm convergence we conclude that

$$
0=\lim \left\|F\left(\mu^{k}\right)-F(\mu)\right\|=\lim \left\|F\left(\xi_{k}\right) \delta_{\gamma}-F(\xi) \delta_{\gamma}\right\|=\lim \left|F\left(\xi_{k}\right)-F(\xi)\right| .
$$

Conversely, given $R>0$ and $\varepsilon>0$ we will find a $\delta>0$, depending on $F$, so that if $\left\|\mu_{j}\right\| \leq R$, $j=1,2$ and $\left\|\mu_{1}-\mu_{2}\right\|<\delta$ then $\left\|F\left(\mu_{1}\right)-F\left(\mu_{2}\right)\right\|<\varepsilon$.

Choose $2=\sup \{|F(\xi):| \xi \mid \leq 1\}$. Choose $\delta_{1}>0$ so that $|\xi| \leq 1,|\eta| \leq 1$, and $|\xi-\eta|<\delta_{1}$ imply $|F(\xi)-F(\eta)| \leq \varepsilon / 4 R$. Let $\delta=\delta_{1} \varepsilon / 4 L$. Then

With $\mu_{j}$ as above let $v=\left|\mu_{1}\right|+\left|\mu_{2}\right|$. Then $\mu_{j}=g_{j} v, g_{j}$ Borel, $\left\|g_{j}\right\|_{L^{\infty}} \leq 1$, and $\|v\| \leq R$.

We then estimate

$$
v\left\{x:\left|g_{1}(x)-g_{2}(x)\right|>\delta_{1}\right\}<\frac{1}{\delta_{1}} \int\left|\mu_{1}-\mu_{2}\right|<\varepsilon / 4 L \text {. }
$$

$$
\begin{aligned}
\left\|F\left(\mu_{1}\right)-F\left(\mu_{2}\right)\right\| & =\int\left|F\left(g_{1}\right)-F\left(g_{2}\right)\right| \mathrm{d} v=\int_{\left|g_{1}-g_{2}\right|>\delta_{1}}+\int_{\left|g_{1}-g_{2}\right|<\delta_{1}} \\
& <2 L v\left\{\left|g_{1}-g_{2}\right|>\delta_{1}\right\}+\frac{\varepsilon}{4 R}\|v\|<\frac{\varepsilon}{2}+\frac{\varepsilon}{2}=\varepsilon .
\end{aligned}
$$

We next turn to the more subtle question of convergence in the weak star topology. The map $\mu \rightarrow F(\mu)$ is not continuous in that topology.

Example 1.10. Let $M([-1,1]: \mathbb{R}) \ni \mu_{k} \equiv \delta_{1 / k}-\delta_{-1 / k}$ and $F(\xi)=|\xi|$, then $\mu_{k}-0$ and $F\left(\mu_{k}\right) \rightarrow 2 \delta_{0} \neq F(0)=0$.

In the scalar case, $d=1$, all homogeneous $F$ are linear combinations of $\xi_{ \pm}$so $(\mu)_{ \pm} \rightarrow(\mu)_{ \pm}$ suffices to insure that $F\left(\mu_{k}\right) \rightarrow F(\mu)$. For $d>1$, vague convergence $\mu_{k} \rightarrow \mu_{k}$ and convergence of the variations $\left(\mu_{k}^{i}\right)_{ \pm} \rightarrow \mu_{ \pm}^{i}$ is not sufficient to guarantee $F\left(\mu_{k}\right) \rightarrow F(\mu)$.

Example 1.11. Let $\Gamma=[-1,1], d=2, \mu_{k}=\left(\delta_{1 / k}, \delta_{2 / k}\right)$, then $\mu_{k} \rightarrow \mu=\left(\delta_{0}, \delta_{0}\right)$ and $\left(\mu_{k}^{i}\right)_{ \pm} \rightarrow\left(\mu^{i}\right)_{ \pm}$. In fact, for any $\eta \in \mathbb{R}^{2},\left(\eta \cdot \mu_{k}\right)_{ \pm} \rightarrow(\eta \cdot \mu)_{ \pm}$. Nevertheless, if $F(\xi)=|\xi|$, then 
$F\left(\mu_{n}\right)=\mu_{n}-2 \delta_{0}$ while $F(\mu)=\sqrt{2} \delta_{0}$. When $F$ is convex there is weak semicontinuity for the $\operatorname{map} \mu \rightarrow F(\mu)$.

Proposition 1.12 [5]. If $F$ is convex then $\mu \rightarrow F(\mu)$ is lower semicontinuous for weak star convergence that is, if $\mu_{k} \rightarrow \mu$ then $F(\mu) \leq \lim F\left(\mu_{k}\right)$.

Weaker conditions than norm convergence suffice to guarantee $F\left(\mu_{k}\right)-F(\mu)$ for all continuous homogeneous $F$.

Example 1.13. If $j \in C_{0}\left(\mathbb{R}^{n}, \mathbb{R}\right), \int j(x) \mathrm{d} x=1, j_{k}(x) \equiv k^{n} j(k x)$, and $\mu \in M\left(\mathbb{R}^{n}, \mathbb{R}^{d}\right) \cap$ $\varepsilon^{\prime}\left(\mathbb{R}^{n}, \mathbb{R}^{d}\right)$ then $\mu_{k} \equiv j_{k} * \mu$ converges vaguely to $\mu$ but not necessarily in norm. However $F\left(\mu_{n}\right) \rightarrow F(\mu)$ for all continuous homogeneous $F$ (see (1.21)). For $\Gamma \subset \mathbb{R}^{n}$ this shows that our definition of $F(\mu)$ is quite natural.

Example 1.14. If one regularizes the components of $\mu$ with different kernels the convergence in example 1.13 may fail. For example if $j \in C_{0}(\mathbb{R}, \mathbb{R}), \int j=1, \operatorname{supp} j \subset(0, \infty), j_{k}(x)=k j(k x)$, $j_{k}(x) \equiv j_{k}(-x), \mu=\left(\delta_{0}, \delta_{0}\right)$, then $\mu_{k} \equiv\left(j_{k} * \delta, j_{2 k} * \delta\right)=\left(j_{k}, j_{2 k}\right) \rightarrow \mu$. However, with $F(\xi)=$ $\left(\xi_{1}^{2}+\xi_{2}^{2}\right)^{1 / 2}, F(\mu)=\sqrt{2} \delta_{0}$, and $F\left(\mu_{k}\right) \rightarrow((\sqrt{5}+1) / 2) \delta_{0}$.

Our main result gives necessary and sufficient conditions on a sequence $\mu_{k} \rightarrow \mu$ in order that $F\left(\mu_{k}\right) \rightarrow F(\mu)$ for all continuous homogeneous $F$.

TheOREM 1.15 . Suppose that $\mu_{k}$ is a sequence in $M\left(\Gamma, \mathbb{R}^{d}\right)$ weak star convergent to $\mu$. Then, the following conditions are equivalent.

(A) For any continuous $F$ positively homogeneous of degree $1, F\left(\mu_{k}\right) \rightarrow F(\mu)$.

(B) $\varphi\left(\mu_{k}\right) \rightarrow \varphi(\mu)$ for one strictly convex norm, $\varphi$, on $\mathbb{R}^{d}$.

(C) For any $\varepsilon>0$ there is an $h \in C\left(\Gamma, \mathbb{R}^{d}\right)$ so that

$$
\varlimsup_{k \rightarrow \infty}\left\|\mu_{k}-h\left|\mu_{k}\right|\right\|<\varepsilon \text {. }
$$

(D) For any $\varepsilon>0$ there is a finite family of disjoint compact subsets $K_{1}, \ldots, K_{N}$ of $\Gamma$ and unit vectors $\xi_{1}, \xi_{2}, \ldots, \xi_{N}$ in $\mathbb{R}^{d}$ such that

$$
\varlimsup_{k \rightarrow \infty}\left\|\mu_{k}-\Sigma \xi_{j} \chi_{K_{j}}\left|\mu_{k}\right|\right\|<\varepsilon .
$$

For condition (B) of the theorem we recall that a norm $\varphi$ on $\mathbb{R}^{d}$ is strictly convex if and only if $(\forall \varepsilon>0)(\exists \delta>0)(\forall \xi, \eta)$

$$
|\xi|=|\eta|=1 \quad \text { and } \quad \varphi((\xi+\eta) / 2))>1-\delta \Rightarrow \varphi(\xi-\eta)<\varepsilon
$$

This result is in fact contained in the proof of theorem 3 of Reschetnyak [10], though it is not enounced in it. But its proof requires many sophisticated notions and lemmas. We give here a very natural and elementary proof of $A \mapsto B$.

The conditions (C) and (D) state in different ways that the "polarization" $\mu_{k} /\left|\mu_{k}\right|$ is nearly independent of $k$.

Example 1.18. $\mu_{k}=\delta_{-1 / k}-\delta_{1 / k} \in M([-1,1]: \mathbb{R})$. Here (A) is violated since $\mu_{k} \rightarrow 0$, $\left|\mu_{k}\right| \rightarrow 2 \delta_{0}$. To see that $(\mathrm{C})$ is violated, notice that for any $h \in C(\mathbb{R}, \mathbb{R})$

$$
\lim \left\|\mu_{k}-h\left|\mu_{k}\right|\right\|=\lim (|1-h(-1 / k)|+|1+h(1 / k)|) \geq 2 \text {. }
$$


Note however that if $h=\chi_{(-\infty, 0)}-\chi_{[0, \infty)}$ then $\mu_{k}=h\left|\mu_{k}\right|$ for all $k$. Thus the continuity of $h$ in (C) is essential. Similarly, if $K_{1}=\left[-1,0\left[, K_{2}=[0,1], \xi_{1}=+1, \xi_{2}=-1\right.\right.$ then $\mu_{k}-$ $\sum_{i} \xi_{i} \chi_{K_{i}}\left|\mu_{k}\right|=0$ for all $k$. Thus the compactness of the $K_{i}$ in condition (D) is essential.

Example 1.19. If $d=1$ and $\mu_{k} \geq 0$ condition (C) is satisfied. One need only take $h=1$. Similarly taking $K=\Gamma$ and $\xi=+1$ we see that (D) is satisfied. The same choices work under the weaker hypothesis $\left\|\left(\mu_{k}\right)_{-}\right\| \rightarrow 0$. An interesting nonnegative example is given by $\mu_{k}=$ $f_{k} \mathrm{~d} x \in M([0,1]: \mathbb{R})$ with

$$
f_{k} \equiv \sum_{0}^{k-1}\left(1+(-1)^{j}\right) \chi_{[j / k,(j+1) / k]}, \quad f \equiv \chi_{[0,1]} .
$$

Then $\mu_{k} \rightarrow \mu \equiv f \mathrm{~d} x$ satisfies the criteria of theorem 1.15 , so $F\left(\mu_{k}\right)=F\left(f_{k}\right) \mathrm{d} x \rightarrow F(\mu)=$ $F(f) \mathrm{d} x$ for $F$ positively homogeneous and continuous. On the other hand, for nonhomogeneous $F$, for example $F(s) \equiv \min \left(s^{2}, 1\right)$ we do not have $F\left(f_{k}\right) \rightarrow F(f)$. This underlines the fact that the set of $F$ in $(A)$ is quite restricted.

Example 1.20. If $\mu_{k} \rightarrow \mu$ in norm let us prove (C) without recourse to theorem 1.15. Let $g$ be in $L^{1}(\Gamma,|\mu|)$ such that $\mu=g|\mu|, \delta>0$ and $h \in C\left(\Gamma, \mathbb{R}^{d}\right)$, so that $\int|h-g||\mu|<\delta / 3$. We may assume that $|h| \leq 1$. Let then $k_{0}$ sufficiently large in order to have for $k \geq k_{0}, \int\left|\mu_{k}-\mu\right|<\delta$. Then for $k \geq k_{0}$,

$$
\int\left|\mu_{k}-h\right| \mu_{k}|| \leq \int\left|\mu_{k}-\mu\right|+\int|\mu-h| \mu||+\int|h|\left|\mu_{k}-\mu\right| \leq 3 \times \delta / 3 .
$$

Similarly, one verifies (D) by approximating $g$ defined by $\mu=g|\mu|$, by a simple function $\Sigma \xi_{i} \chi_{K_{i}}$ with $K_{i}$ compact and unit vectors $\xi_{i}$.

Example 1.21. If $\mu \in M\left(\mathbb{R}^{n}, \mathbb{R}^{d}\right)$ has compact support and $\mu_{k}=j_{k} * \mu$ as in example 1.13 we show that (C) is satisfied. For any $\varepsilon>0$, choose $h \in C_{0}\left(\mathbb{R}^{n}, \mathbb{R}^{d}\right),\|h\|_{L^{\infty}} \leq 1$ such that $\|\mu-h|\mu|\|<\varepsilon / 6$. Then choose $N>0$ so that for $k \geq N$,

$$
x-y \in \operatorname{supp} j_{k} \Rightarrow|h(x)-h(y)|<\varepsilon\|\mu\| / 6 .
$$

We use two estimates concerning convolution with $j_{k}$ : for any any finite $\nu \in M\left(\mathbb{R}^{n}, \mathbb{R}^{d}\right)$

$$
\begin{gathered}
\left\|j_{k} * v\right\| \leq\|v\|, \quad \text { and for } n \geq N \\
\left\|j_{k} *(h v)-h\left(j_{k} * v\right)\right\|<\varepsilon\|v\| / 6 .
\end{gathered}
$$

The latter follows on expressing the left hand side as

$$
\int\left|\int j_{k}(x-y)(h(x)-h(y)) \mathrm{d} v(y)\right| \mathrm{d} x .
$$

Using these estimates yields for $n \geq N$

$$
\begin{aligned}
\mu_{k} & \equiv j_{k} * \mu=j_{k} *(h|\mu|)+v_{1}, \quad\left\|v_{1}\right\|<\varepsilon / 6 \\
& =h\left(j_{k} *(|\mu|)+v_{2}+v_{1}, \quad\left\|v_{2}\right\|<\varepsilon / 6 .\right.
\end{aligned}
$$


Then, since $j_{k} *|\mu| \geq 0$,

$$
\begin{aligned}
\left|\mu_{k}\right| & =|h|\left(j_{k} *|\mu|\right)+v_{3}, \quad\left\|v_{3}\right\|<2 \varepsilon / 6 . \\
& =j_{k} *(|h||\mu|)+v_{4}+v_{3}, \quad\left\|v_{4}\right\|<\varepsilon / 6 \\
& =j_{k}+(|\mu|)+v_{5}+v_{4}+v_{3}, \quad\left\|v_{5}\right\|<\varepsilon / 6 .
\end{aligned}
$$

Since $\mu_{k}-h\left|\mu_{k}\right|=v_{1}+v_{2}+h\left(v_{3}+v_{4}+v_{5}\right)$, we have for $n \geq N,\left\|\mu_{k}-h\left|\mu_{k}\right|\right\|<\varepsilon$.

To prove theorem 1.15 we will show $\mathrm{A} \Rightarrow \mathrm{B} \Rightarrow \mathrm{C} \Rightarrow \mathrm{D} \Rightarrow \mathrm{A}$. The implication $\mathrm{A} \Rightarrow \mathrm{B}$ is trivial.

Proof that $(\mathrm{B}) \Rightarrow(\mathrm{C})$. Choose $\Lambda \in\left[1, \infty\left[\right.\right.$ so that $\forall \xi, \eta \in \mathbb{R}^{d}$,

$$
\Lambda^{-1 / 2}|\xi-\eta| \leq \varphi(\xi-\eta) \leq \Lambda^{1 / 2}|\xi-\eta| .
$$

Then given any $\varepsilon>0$ we choose $\delta>0$ so that

$$
(\varphi(\xi)=\varphi(\eta)=1 \quad \text { and } \quad \varphi((\xi+\eta) / 2) \geq 1-\delta) \Rightarrow \varphi(\xi-\eta)<\varepsilon /\left(\Lambda \sup _{k}\left\|\varphi\left(\mu_{k}\right)\right\|_{2}\right) .
$$

Since $\mu_{k} \ll \varphi\left(\mu_{k}\right)$ and $\left.\mu \ll \varphi / \mu\right)$ we may choose Borel measureable functions $g_{k}$ and $g$ so that $\mu_{k}=g_{k} \varphi\left(\mu_{k}\right), \mu=g \varphi(\mu)$ and $\varphi\left(g_{k}\right)=\varphi(g)=1$ at all points of $\Gamma$.

Choose $h \in C\left(\Gamma, \mathbb{R}^{d}\right)$ with $\varphi(h) \equiv 1$ and

$$
\int \varphi(h-g) \mathrm{d} \varphi(\mu)<\varepsilon \delta / 8 \Lambda .
$$

Since $\left\langle\varphi\left(\mu_{k}\right), 1\right\rangle \rightarrow\langle\varphi(\mu), 1\rangle$ we may choose $k_{0}$ so that for all $k>k_{0}$

$$
\int \mathrm{d} \varphi\left(\mu_{k}\right) \leq \int \mathrm{d} \varphi(\mu)+\varepsilon \delta / 8 \Lambda .
$$

The subadditivity of $\varphi$ shows that

$$
1=\varphi(h) \leq \varphi\left(\frac{g+h}{2}\right)+\varphi\left(\frac{h-g}{2}\right) .
$$

Since $\int \varphi(h-g) \mathrm{d} \varphi(\mu)<\varepsilon \delta / 8 \Lambda$, we find

$$
\begin{aligned}
\int \mathrm{d} \varphi\left(\mu_{k}\right) & \leq \int\left(\varphi\left(\frac{g+h}{2}\right)+\varphi\left(\frac{h-g}{2}\right)\right) \mathrm{d} \varphi(\mu)+\varepsilon \delta / 8 \Lambda \\
& =\int \varphi\left(\frac{g+h}{2}\right) \mathrm{d} \varphi(\mu)+3 \varepsilon \delta / 16 \Lambda \\
& =\frac{1}{2} \int \mathrm{d} \varphi(\mu+h \varphi(\mu))+3 \varepsilon \delta / 16 \Lambda .
\end{aligned}
$$

Since $\mu_{k}+h \varphi\left(\mu_{k}\right) \rightarrow \mu+h \varphi(\mu)$, the lower semicontinuity (1.12) implies that we may choose $k_{1} \geq k_{0}$ so that for all $k>k_{1}$,

$$
\int \mathrm{d} \varphi(\mu+h \varphi(\mu)) \leq \int \mathrm{d} \varphi\left(\mu_{k}+h \varphi\left(\mu_{k}\right)\right)+\frac{\varepsilon \delta}{8 \Lambda} .
$$


Then,

Rewriting yields

$$
\begin{aligned}
\int \mathrm{d} \varphi\left(\mu_{k}\right) & \leq \frac{1}{2} \int \mathrm{d} \varphi\left(\mu_{k}+h \varphi\left(\mu_{k}\right)\right)+\varepsilon \delta / 4 \Lambda \\
& =\int \varphi\left(\frac{g_{k}+h}{2}\right) \mathrm{d} \varphi\left(\mu_{k}\right)+\varepsilon \delta / 4 \Lambda
\end{aligned}
$$

$$
\int\left(1-\varphi\left(\left(g_{k}+h\right) / 2\right)\right) \mathrm{d} \varphi\left(\mu_{k}\right) \leq \varepsilon \delta / 4 \Lambda .
$$

Let $A_{k} \equiv\left\{x \in \Gamma: \varphi\left(\left(g_{k}(x)+h(x)\right) / 2\right)<1-\delta\right\}$. Then $\varphi\left(\mu_{k}\right)\left(A_{k}\right)<\varepsilon / 4 \Lambda$ and on $\Gamma \backslash A_{k}$,

$$
\varphi\left(g_{\mathrm{k}}-h\right)<\varepsilon /\left(2 \Lambda \sup \left\|\varphi\left(\mu_{k}\right)\right\|\right) \text {. }
$$

To estimate $\left\|\mu_{k}-h\left|\mu_{k}\right|\right\|$ begin with

$$
\begin{aligned}
\left\|\mu_{k}-h\left|\mu_{k}\right|\right\| & =\int\left|g_{k}-h\right| \mathrm{d}\left|\mu_{k}\right| \leq \Lambda^{1 / 2} \int \varphi\left(g_{k}-h\right) \mathrm{d}\left|\mu_{k}\right| \\
& \leq \Lambda \int_{\Gamma} \varphi\left(g_{k}-h\right) \mathrm{d} \varphi\left(\mu_{k}\right) .
\end{aligned}
$$

The estimate for $\varphi\left(g_{k}-h\right)$ on $\Gamma \backslash A_{k}$ shows that the contribution from $\Gamma \backslash A_{k}$ is dominated by $\varepsilon / 2$. On $A_{k}$ use $\varphi\left(g_{k}-h\right) \leq 2$ together with the estimate for the size of $A_{k}$ to show that the contribution from $A_{k}$ is also bounded by $\varepsilon / 2$. Thus, for $k>k_{1}$, we have $\left\|u_{k}-h\left|\mu_{k}\right|\right\|<\varepsilon$ and the proof of $(C)$ is complete.

Proof that $(\mathrm{C}) \Rightarrow(\mathrm{D})$. Given $\varepsilon>0$ choose $h_{0} \in C\left(\Gamma, \mathbb{R}^{d}\right)$ so that $\varlimsup i m\left\|\mu_{k}-h_{0}\left|\mu_{k}\right|\right\|<\varepsilon^{2}$. Let

$$
h(x)= \begin{cases}h_{0}(x) & \text { if }\left|h_{0}(x)\right| \leq 1 \\ h_{0}(x) /\left|h_{0}(x)\right| & \text { if }\left|h_{0}(x)\right|>1 .\end{cases}
$$

Then $h \in C\left(\Gamma, \mathbb{R}^{d}\right)$, and $\|h\|_{L^{\infty}} \leq 1$. For $k \geq 1$, choose Borel measurable $h_{k}$ with $\mu_{k}=h_{k}\left|\mu_{k}\right|$, and $\left|h_{k}(x)\right| \leq 1$ for all $x \in \Gamma$. Then since $\left|h_{k}\right| \leq 1$,

$$
\begin{aligned}
\left\|\mu_{k}-h\left|\mu_{k}\right|\right\| & =\int\left|h_{k}-h\right| \mathrm{d}\left|\mu_{k}\right| \leq \int\left|h_{k}-h_{0}\right| \mathrm{d}\left|\mu_{k}\right| \\
& =\left\|\mu_{k}-h_{0}\left|\mu_{k}\right|\right\| .
\end{aligned}
$$

Thus $\overline{\lim }\left\|\mu_{k}-h\left|\mu_{k}\right|\right\|<\varepsilon^{2}$. It follows that

$$
\left|\mu_{k}\right|\{x:|h(x)| \leq 1-\varepsilon\}<\varepsilon .
$$

Let $A=\left\{x \in \mathbb{R}^{d}: 1-\varepsilon \leq|x| \leq 1\right\}$. Pave $\mathbb{R}^{d}$ by a family of hyperplanes parallel to the axes so that

(1) the rectangles of the paving have diameter less than $\varepsilon$, and

(2) for each hyperplane $H$ defining the paving,

$$
|\mu|\left(h^{-1}(H)\right)=0=\left|\mu_{k}\right|\left(h^{-1}(H)\right), \quad k=1,2, \ldots
$$


Since only a countable number of hyperplanes have inverse image by $h$ of positive measure for $\mu$ or some $\mu_{k}$, pavings satisfying (1) and (2) are easy to find.

Let $R_{1}, R_{2}, \ldots, R_{N}$ be the closure of the rectangles of the paving with nonvoid intersection with $A$, Let $E_{i} \equiv h^{-1}\left(\operatorname{Int}\left(R_{i}\right)\right), i=1,2, \ldots, N$. Then the $E_{i}$ are open in $\Gamma$ and $\partial E_{i}$, contained in $h^{-1}$ of the hyperplanes in the paving, are null sets for $|\mu|$ and $\left|\mu_{k}\right|$. As

we have

$$
\left.\Gamma \backslash U E_{i} \subset\{|h|<1-\varepsilon\} \cup U \partial E_{i}\right\},
$$

$$
\left|\mu_{k}\right|\left(\Gamma \backslash U E_{i}\right)<\varepsilon
$$

For each $i$, choose a compact set $\gamma_{i} \subset E_{i}$ with

$$
|\mu|\left(E_{i} \backslash \gamma_{i}\right)<\varepsilon / N
$$

Choose open sets $\Omega_{i}^{1}, \Omega_{i}^{2}$ with $\gamma_{i} \subset \Omega_{i}^{1} \subset \subset \Omega_{i}^{2} \subset \subset E_{i}$ and let $K_{i} \equiv\left(E_{i} \backslash \Omega^{2}\right) \cup \Omega_{i}^{1}$.

As $h\left(E_{i}\right)$ is a rectangle of diameter less than $\varepsilon$ which intersects $A$ we can choose a unit vector $\xi_{i} \in \mathbb{R}^{d}$ with $\left|h(x)-\xi_{i}\right| \leq 2 \varepsilon$ for all $x \in E_{i}$.

We will show that these choices of $K_{i}, \xi_{i}$ serve to verify (D). On $U K_{i}$ we have $\left|h-\Sigma_{i} \xi_{i} \chi_{K_{i}}\right|<2 \varepsilon$, and on $\Gamma \backslash U k_{i},\left|h-\Sigma \xi_{i} \chi_{K_{i}}\right| \leq 2$ so

$$
\left\|\left(h-\Sigma \xi_{i} \chi_{K_{i}}\right)\left|\mu_{k}\right|\right\| \leq 2 \varepsilon\left\|\mu_{k}\right\|+2\left|\mu_{k}\right|\left(\Gamma \backslash U K_{i}\right) .
$$

Write $\Gamma \backslash U K_{i}=\left(\Gamma \backslash U E_{i}\right) \cup U\left(E_{i} \backslash K_{i}\right)$. The first set has $\left|\mu_{k}\right|$ measure less than $\varepsilon$. For the second, notice that $\left(E_{i} \backslash K_{i}\right) \subset \subset E_{i} \backslash \gamma_{i}$ so we may choose $\varphi_{i} \in C_{0}\left(E_{i} \backslash \gamma_{i}, \mathbb{R}\right)$ with $0 \leq \varphi_{i} \leq 1 \varphi_{i}=1$ on $E_{i} \backslash K_{i}$. We then have

$$
\left\|\left(h-\Sigma \xi_{i} \chi_{K_{i}}\right)\left|\mu_{k}\right|\right\| \leq 2 \varepsilon\left\|\mu_{k}\right\|+2 \varepsilon+\left\langle\left|\mu_{k}\right|, \sum_{i} \varphi_{i}\right\rangle .
$$

To estimate the last term, note that $E_{i}=h^{-1}\left(\right.$ Int $\left.R_{i}\right)$ so $|h|^{2} \geq 1-2 \varepsilon$ on $E_{i}$. Taking the scalar product of $\mu_{k}-h\left|\mu_{k}\right|$ with $h$ yields

$$
\left\|\left(h \cdot \mu_{k}-\left|\mu_{k}\right|\right) \chi_{U E_{i}}\right\|<\varepsilon^{2}+2 \varepsilon\left\|\mu_{k}\right\| .
$$

The supports of $\varphi_{i}$ are disjoint and contained in $E_{i}$ so $\left\|\Sigma \varphi_{i}\right\|_{L^{\infty}} \leq 1$. Thus,

Now,

$$
\left|\left\langle\left|\mu_{k}\right|, \Sigma \varphi_{i}\right\rangle-\left\langle h \cdot \mu_{k}, \Sigma \varphi_{i}\right\rangle\right|<\varepsilon^{2}+2 \varepsilon\left\|\mu_{k}\right\| \text {. }
$$

$$
\left\langle h \cdot \mu_{k}, \Sigma \varphi_{i}\right\rangle=\left\langle\mu_{k}, h \Sigma \varphi_{i}\right\rangle \rightarrow\left\langle\mu, h \Sigma \varphi_{i}\right\rangle \leq|\mu|\left(U E_{i} \backslash \gamma_{i}\right)<\varepsilon
$$

the last estimate following from (1.22). Thus

$$
\varlimsup_{k}\left\|h-\Sigma \xi_{i} \chi_{K_{i}}\left|\mu_{k}\right|\right\| \leq\left(3+4 \sup _{k}\left\|\mu_{k}\right\|\right) \varepsilon+\varepsilon^{2}
$$

and $(\mathrm{D})$ is proved.

Proof that (D) $\Rightarrow$ (A). Choose $R>\sup \left\|\mu_{k}\right\|$. Given $\varepsilon>0$, part 3 of theorem 1.7 shows that there is a $\delta \in] 0, \varepsilon]$ so that if $v_{1}, v_{2} \in M\left(\Gamma, \mathbb{R}^{d}\right),\left\|v_{1}\right\| \leq R,\left\|v_{1}-v_{2}\right\|<3 \delta$ then $\left\|F\left(v_{1}\right)-F\left(v_{2}\right)\right\|<\varepsilon$.

Choose $K_{i}, \xi_{i}$ as in (D) so that

$$
\varlimsup\left\|\mu_{k}-\Sigma \xi_{i} \chi_{K_{i}}\left|\mu_{k}\right|\right\| \leq \delta
$$


Then $\Gamma \backslash U K_{i}$ is an open subset of $\Gamma$ with

It follows that

$$
\widetilde{\lim }\left|\mu_{k}\right|\left(\Gamma \backslash U K_{i}\right) \leq \delta
$$

$$
|\mu|\left(\Gamma \backslash U K_{i}\right) \leq \delta .
$$

Choose $\rho_{i} \in C(\Gamma, \mathbb{R}), 0 \leq \rho_{i} \leq 1$ so that $\rho_{i}=1$ on $K_{i}$ and for $i \neq j, \rho_{i} \rho_{j}=0$. Let $\rho=\Sigma \rho_{i}$. Then since $\|\rho\| \leq 1,\left|\mu_{k}\right|-\rho \cdot \mu_{k}$ is a positive measure. Then (1.23) and (1.17) yield

$$
\varlimsup\left\|\left|\mu_{k}\right|-\rho \cdot \mu_{k}\right\|<2 \delta \quad \text { and } \quad \varlimsup \lim \left\|\mu_{k}-\rho\left|\mu_{k}\right|\right\|<2 \delta \text {. }
$$

For any $\varphi \in C(\Gamma, \mathbb{R})$ with $0 \leq \varphi \leq 1$

$$
\langle|\mu|, \varphi) \leq \lim \left\langle\left|\mu_{k}\right|, \varphi\right\rangle \leq \lim \left\langle\rho \cdot \mu_{k}, \varphi\right\rangle+2 \delta=\langle\rho \cdot \mu, \varphi\rangle+2 \delta .
$$

Thus, $0 \leq\langle|\mu|-\rho \cdot \mu, \varphi\rangle \leq 2 \delta$, and we conclude that $\||\mu|-\rho \cdot \mu\| \leq 2 \delta$. Then,

$$
\left|\mu_{k}\right|-|\mu|=\left(\left|\mu_{k}\right|-\rho \cdot \mu_{k}\right)+\left(\rho \cdot \mu_{k}-\rho \cdot \mu\right)+(\rho \cdot \mu-|\mu|) .
$$

The first and last measure have norm less than $2 \delta$ and the middle converges vaguely to zero. Thus for any $\gamma \in C(K, \mathbb{R}),\|\gamma\|_{L^{\infty}} \leq 1$

$$
\left.\varlimsup \lim _{k}\left|\mu_{k}\right|-|\mu|, \gamma\right\rangle \leq 4 \delta \text {. }
$$

As $\delta \geq \varepsilon$ and $\varepsilon$ was arbitrary we conclude that $\left|\mu_{k}\right| \rightarrow|\mu|$. Passing to the limit in (1.24) yields $\|\mu-\rho|\mu|\| \leq 2 \delta$. Thus, the choice of $\delta$ yields,

and

$$
\varlimsup \lim \left\|F\left(\mu_{k}\right)-F(\rho)\left|\mu_{k}\right|\right\| \leq \varepsilon,
$$

Thus,

$$
\|F(\mu)-F(\rho)|\mu|\| \leq \varepsilon .
$$

$$
F\left(\mu_{k}\right)-F(\mu)=F(\rho)\left(\left|\mu_{k}\right|-|\mu|\right)+v_{k}
$$

where the first term tends vaguely to zero and $\varlimsup \lim \left\|v_{k}\right\| \leq 2 \varepsilon$. Since $\varepsilon>0$ was arbitrary, it follows that $F\left(\mu_{k}\right) \rightarrow F(\mu)$.

We end this section by noting that theorem 1.15, though given on a compact set $\Gamma$ applies to yicld corresponding results for measures defined on an open subset $\Omega \subset \mathbb{R}^{d}$. Four natural choices present themselves, the duals of $C_{0}\left(\Omega, \mathbb{R}^{d}\right) \subset \dot{C}\left(\Omega: \mathbb{R}^{d}\right) \equiv\left\{u \in C\left(\Omega: \mathbb{R}^{d}\right): u=0\right.$ on $\partial \Omega\} \subset C\left(\Omega: \mathbb{R}^{d}\right) \subset B C\left(\Omega, \mathbb{R}^{d}\right) \equiv C\left(\Omega: \mathbb{R}^{d}\right) \cap L^{\infty}\left(\Omega: \mathbb{R}^{d}\right)$. The first is the set of continuous functions with compact support. The topology is the usual inductive limit. The last three are Banach spaces with the sup norm. In all four cases weak star convergence of measures is described in terms of weak star convergence in $C\left(\Gamma: \mathbb{R}^{d}\right)^{\prime}$ for suitable compact subsets $\Gamma$ of $\Omega$. We have

$$
\mu_{k} \rightarrow \mu \text { in } C_{0}\left(\Omega: \mathbb{R}^{h}\right)^{\prime} \quad \text { iff } \forall_{\Gamma \subset \Omega, \text { compact }}, \mu_{k} \rightarrow \mu \text { in } C\left(\Gamma: \mathbb{R}^{h}\right)^{\prime}
$$

(II) $\quad \mu_{k} \rightarrow \mu$ in $B C\left(\Omega: \mathbb{R}^{h}\right)^{\prime} \quad$ if $(1.25)$ holds and $\forall_{\delta>0}, \exists_{\Gamma \subset \Omega \text {, compact }},|\mu|(\Omega \backslash \Gamma)<\delta$.

Finally, $C(\Omega)$ and $\stackrel{\circ}{C}(\Omega)^{\prime}$ are the same as $B C(\Omega)^{\prime}$, and, weak star convergence in each of these spaces is equivalent to weak star convergence in each of the others. The next corollaries are then direct consequences of theorem 1.15 . 
CoRollaRy 1.27 . If $\Omega \subset \mathbb{R}^{n}$ is open and $\mu_{k} \rightarrow \mu$ in the weak star topology for $C_{0}\left(\Omega: \mathbb{R}^{d}\right)^{\prime}$ then the following conditions are equivalent.

(A) $F\left(\mu_{k}\right) \rightarrow F(\mu)$ for all continuous $F$ positively homogeneous of degree 1 .

(B) $\varphi\left(\mu_{k}\right)-\varphi(\mu)$ for one strictly convex norm $\varphi$ on $\mathbb{R}^{d}$.

(C) For any compact $\Gamma \subset \Omega$ and $\varepsilon>0$ there is an $h \in C\left(\Gamma, \mathbb{R}^{d}\right)$ so that $\overline{\lim }\left\|\mu_{k}-h\left|\mu_{k}\right|\right\|<\varepsilon$.

(D) For any compact $\Gamma \subset \Omega$ and $\varepsilon>0$ there is a finite disjoint family of compact sets $K_{i} \subset \Gamma$ and unit vectors $\xi_{i} \in \mathbb{R}^{d}$ with $\varlimsup \lim \left\|\mu_{k}-\Sigma \xi_{i} \chi_{K_{i}}\left|\mu_{k}\right|\right\|<\varepsilon$.

CoRollaRY 1.28 . If $\Omega \subset \mathbb{R}^{n}$ is open and $\mu_{k} \rightarrow \mu$ in the weak star topology for $B C\left(\Omega: \mathbb{R}^{d}\right)^{\prime}$, then the following conditions are equivalent:

(a) $F\left(\mu_{k}\right) \rightarrow F(\mu)$ in $B C^{\prime}$ for all continuous $F$ positively homogeneous of degree 1 .

(b) $\varphi\left(\mu_{k}\right) \rightarrow \varphi(\mu)$ in $B C^{\prime}$ for one strictly convex norm $\varphi$ on $\mathbb{R}^{d}$.

(c) For any $\varepsilon>0$ there is an $h \in C_{0}\left(\Omega: \mathbb{R}^{d}\right)$ so that $\overline{\lim }\left\|\mu_{k}-h\left|\mu_{k}\right|\right\|<\varepsilon$.

(d) For any $\varepsilon>0$ there is a finite disjoint family of compact subsets $K_{i} \subset \Omega$ and unit vectors $\xi_{i} \in \mathbb{R}^{d}$ with $\varlimsup i m\left\|\mu_{k}-\Sigma \xi_{i} \chi_{K_{j}}\left|\mu_{k}\right|\right\|<\varepsilon$.

The proof of the corollaries are left to the reader.

\section{2. $f(\mu)$ WITH $f$ SUBLINEAR}

Definition. $f: \mathbb{R}^{d} \rightarrow \mathbb{R}$ is called sublinear if $\lim _{|\xi| \rightarrow \infty} f(\xi) /|\xi|=0$, that is for any $\varepsilon>0$ there is an $R$ so that for $|\xi|>R, f(\xi) /|\xi|<\varepsilon$.

Example 2.1. $f_{\delta}(\xi)=|\xi| /\left(1+\delta|\xi|^{2}\right)$ and the components $f_{N}^{i}$ of $f_{N}=\left(f_{N}^{1}, \ldots, f_{N}^{d}\right)$,

$$
f_{N}(\xi)= \begin{cases}\xi & \text { if }|\xi| \leq N \\ N \xi /|\xi| & \text { if }|\xi|>N\end{cases}
$$

are all continuous sublinear functions.

Definition. Suppose $\Gamma \subset \mathbb{R}^{n}$ is compact and $\mathrm{d} x$ is Lebesque measure. For $\mu \in M\left(\Gamma, \mathbb{R}^{d}\right)$ and sublinear $f \in C\left(\mathbb{R}^{d}, \mathbb{R}\right)$ with $f(0)=0$ the measure $f(\mu)$ is defined as $f \circ g \mathrm{~d} x$ where $\mu=g \mathrm{~d} x+v$ is the Lebesque decomposition of $\mu$ with $g: \Gamma \rightarrow \mathbb{R}^{d}$ Borel measureable and integrable $\mathrm{d} x$.

Remark. The normalization of Lebesque measure is critical. If one replaces $\mathrm{d} x$ by $a \mathrm{~d} x$, $a \in] 0, \infty[$ then the Lebesque decomposition becomes $(g / a)(a \mathrm{~d} x)+v$ and one would get $a f(g / a) \mathrm{d} x$, a different result.

Properties 2.2. (i) $f(\mu) \ll \mathrm{d} x$. (ii) If $\mu \perp \mathrm{d} x$ then $f(\mu)=0$. (iii) If $\left(\mu_{1}-\mu_{2}\right) \perp \mathrm{d} x$ then $f\left(\mu_{1}\right)=f\left(\mu_{2}\right)$. (iv) If $M=\|f(\xi) /|\xi|\|_{L^{\infty}\left(\mathbb{R}^{d}\right)}<\infty$ then $\|f(\mu)\| \leq M\|\mu\|$.

$$
\text { If } \mu_{k}=j_{k} * \mu \text { as in (1.13) then } f\left(\mu_{k}\right) \rightarrow f(\mu) \text {. }
$$

This follows from Lebesque's Differentiation theorem as we show in example 2.6. Property (2.3) shows that the definition of $f(\mu)$ is natural. If one tried the same definition, $f(\mu)=f(g) \mathrm{d} x$, for $f$ of linear growth, $|f(x)| \leq c|x|$, the continuity property (2.3) would no longer be true.

The analog of theorem 1.15 for sublinear functions is the following. 
THEOREM 2.4. Suppose $\Gamma$ is a compact subset of $\mathbb{R}^{n}$ and that $\mu_{k}, \mu \in M\left(\Gamma, \mathbb{R}^{d}\right)$ have Lebesque decompositions $\mu_{k}=g_{k} \mathrm{~d} x+v_{k}, \mu=g \mathrm{~d} x+v$ with respect to Lebesque measure $\mathrm{d} x$. Then the following conditions are equivalent.

$(\alpha)$ For any sublinear $f \in C\left(\mathbb{R}^{d}, \mathbb{R}\right), f\left(\mu_{k}\right) \rightarrow f(\mu)$.

(B) For any sublinear $f \in C\left(\mathbb{R}^{d}, \mathbb{R}\right), f\left(\mu_{k}\right) \rightarrow f(\mu)$.

$(\gamma)$ The functions $g_{k}$ are bounded in $L^{1}\left(\Gamma, \mathbb{R}^{d}, \mathrm{~d} x\right)$ and $g_{k}$ converges to $g$ in measure, that is, for any $\eta>0$

$$
\varlimsup \mathrm{\operatorname {lim }} \mathrm{d} x\left\{x \in \Gamma:\left|g(x)-g_{k}(x)\right|>\eta\right\}=0
$$

Example 2.6. We verify that $\mu_{k}=j_{k} * \mu$ as in example 1.13 satisfy $(\gamma)$. First $\mu_{k} \ll \mathrm{d} x$ and $\left\|\mu_{k}\right\| \leq\|j\|_{L^{1}\left(\mathbb{R}^{d}, \mathrm{~d} x\right)}\|\mu\|$, shows the boundedness of the $g_{k}=\left(j_{k} * g\right)+j_{k} * v$. It is classical that $\left\|j_{k} * g-g\right\|_{L^{1}\left(\mathbb{R}^{d}, \mathrm{~d} x\right)} \rightarrow 0$ as $k \rightarrow 0$. Thus it suffices to remark that $j_{k} * v$ converges to zero in measure, a consequence of Lebesque's Differentiation theorem. In contrast to example 1.14 one may regularize the components $\mu^{i}$ with different kernels and the above argument shows that ( $\gamma$ ) remains valid, so $f\left(\mu_{k}\right) \rightarrow f(\mu)$.

Example 2.7. The condition of theorem 1.15 are satisfied by $\mu_{k}=\sum_{j=0}^{k-1} \delta_{j / k} / k \rightarrow \chi_{[0,1]} \mathrm{d} x$, since $\mu_{k} \geq 0$ (see example 1.19). Here $g_{k}=0$ and $g=\chi_{[0,1]} \mathrm{d} x$, since $\mu_{k} \geq 0$ (see example 1.19). Here $g_{k}=0$ and $g=\chi_{[0,1]}$ so the conditions of theorem 2.4 are violated. On the other hand, $\mu_{k}=\sum_{j=0}^{k-1}(-1)^{j} \delta_{j / k} / k \rightarrow 0$ satisfies the hypotheses of theorem (2.3) since $0=g_{k} \rightarrow g=0$ but violates the hypotheses of theorem 1.15 .

To prove theorem 2.4 we will show that $(\alpha) \Rightarrow(\gamma) \Rightarrow(\beta)$. Since it is clear that $(\beta) \Rightarrow(\alpha)$, this is sufficient.

Proof that $(\alpha) \Rightarrow(\gamma)$. Let us consider the following subspace $C\left(\mathbb{R}^{d}, \mathbb{R}\right)$ :

$$
S=\left\{f \in C\left(\mathbb{R}^{d}, \mathbb{R}\right) \lim _{|X| \rightarrow+\infty} \frac{f(X)}{|X|+1}=0\right\} .
$$

Endowed with the norm $\operatorname{Sup}_{X \in \mathbb{R}^{d}}(\|f(X)\| /(|X|+1)), S$ is a Banach space. Let us consider the following sequence of continuous linear forms:

$$
\begin{aligned}
T_{n}: S & \rightarrow \mathbb{R} \\
f & \rightarrow \int_{\Omega} f\left(g_{n}\right) .
\end{aligned}
$$

It is easy to see that $T_{n}$ is continuous for the previous norms and that $\left\|T_{n}\right\|=\int_{\Gamma}\left(\left|g_{n}\right|+1\right)$. Since for every $f \in S, T_{n}(f) \rightarrow T(f)$ where $T(f)=\int_{\Gamma} f(g)$, we obtain by the Banach Steinhaus theorem that

which implies that

$$
\widetilde{\lim }\left\|T_{n}\right\| \leq+\infty
$$

$$
\int_{\Omega}\left|g_{n}\right| \leq M<+\infty \text {. }
$$


So the $g_{k}$ are bounded in $L^{1}(\Gamma, \mathrm{d} x)$. Given $\varepsilon>0$ choose $N>4 \int|g| \mathrm{d} x / \varepsilon$. Choose $k_{0}>0$ so that $k>k_{0}$ implies $\int\left|g_{k}\right| \mathrm{d} x<2 \int|g| \mathrm{d} x$. Then for $k>k_{0}$

and

$$
\mathrm{d} x\left\{x:\left|g_{k}(x)\right|>N\right\}<2 \frac{\int|g| \mathrm{d} x}{N}<\varepsilon / 2,
$$

$$
\mathrm{d} x\{x:|g(x)|>N\}<\frac{\int|g| \mathrm{d} x}{N}<\varepsilon / 4 .
$$

Let $f_{N}^{i} \in C\left(\mathbb{R}^{d}, \mathbb{R}\right)$ be as in (2.1) and define $\varphi \in C(\mathbb{R}, \mathbb{R})$ by

$$
\varphi(s)= \begin{cases}s^{2} & \text { if }|s| \leq N \\ N^{2} & \text { if }|s| \geq N .\end{cases}
$$

Since $f_{N}^{i}$ and $\varphi \circ f_{N}^{i}$ are sublinear and continuous $l_{k}^{i} \equiv f_{N}^{i}\left(g_{k}\right), l^{i} \equiv f^{i}(g)$ satisfy

$$
\begin{aligned}
l_{k}^{i} \mathrm{~d} x & \rightarrow l^{i} \mathrm{~d} x \\
\left(l_{k}^{i}\right)^{2} \mathrm{~d} x & \rightarrow\left(l^{i}\right)^{2} \mathrm{~d} x .
\end{aligned}
$$

Then

$$
\int\left|f_{N}(g)-f_{N}\left(g_{k}\right)\right|^{2} \mathrm{~d} x=\sum_{i} \int\left(l_{k}^{i}\right)^{2}+\left(l^{i}\right)^{2} \mathrm{~d} x-\sum_{i} \int 2 l_{k}^{i} l^{i} \mathrm{~d} x .
$$

Using (2.11) for the first sum and (2.10) for the second shows that the right hand side converges to

$$
\sum_{i} \int 2\left(l^{i}\right)^{2} \mathrm{~d} x-\sum_{i} \int 2\left(l^{i}\right)^{2} \mathrm{~d} x=0 .
$$

Since $f_{N}\left(g_{k}\right)=g_{k}$ when $\left|g_{k}\right| \leq N$ and $f_{N}(g)=g$ when $|g| \leq N$ we see that we may choose $K$ so that $k \geq K$ implies

$$
\mathrm{d} x\left\{x:\left|g_{k}(x)\right| \leq N \text { and }|g(x)| \leq N \text { and }\left|g_{k}(x)-g(x)\right| \geq \eta\right\}<\varepsilon / 4 .
$$

This combined with (2.8) and (2.9) proved that

$$
\varlimsup \mathrm{lim} \mathrm{d} x\left\{x:\left|g_{k}(x)-g(x)\right| \geq \eta\right\}<\varepsilon .
$$

As $\varepsilon>0$ was arbitrary this proves the desired result, (2.5).

Proof that $(\gamma) \Rightarrow(\beta)$. Let us remark to begin with that the image by $f$ sublinear of a bounded set in $L^{1}$ is equi-integrable. In other words, $\forall L>0, \forall \varepsilon>0$,

$$
\text { उ } \delta>0, \quad \text { meas } A<\delta \Rightarrow \operatorname{Sup}_{\gamma \in L^{L}, \delta|g| \leq L}\left\{\int_{A}|f(g)|\right\}<\varepsilon .
$$

Indeed let $R>0$ be so that $|\xi|>R \Rightarrow|f(\xi)| \leq \varepsilon|\xi| / 2 L$, and let $\delta=\varepsilon /\left(2 \sup _{B(x)}|f|+1\right)$; then for every $g$ in $L^{1}$, so that $\int|g| \leq L$, and for every $A$ such that meas $A<\delta$,

$$
\begin{aligned}
\int_{A} f(g(x)) \mathrm{d} x & \leq \int_{A \cap\{x,|g(x)|>R\}} f(g(x)) \mathrm{d} x+\int_{A \cap\{x,|g(x)| \leq R\}} f(g(x)) \mathrm{d} x \\
& \leq \frac{\varepsilon}{2 L} \int_{A}|g(x)|+\operatorname{Sup}_{B(0, R)}|f| \operatorname{meas}[A\} \leq \frac{\varepsilon}{2}+\frac{\varepsilon}{2}=\varepsilon .
\end{aligned}
$$


Let then $L=\sup \left(\int\left|g_{k} \mathrm{~d} x\right|+\int|g \mathrm{~d} x|\right)<\infty$. Given $\varepsilon>0$, let $R$ be so that for $|\xi| \geq R,|f(\xi)| \leq$ $\varepsilon|\xi| / 4 L$. Choose $\delta>0, \delta<R$, so that for $x$ and $y$ in the ball of radius $2 R$ centered at $0 \in \mathbb{R}^{d}$

$$
|x-y|<\delta \Rightarrow|f(x)-f(y)|<\varepsilon / 4 \mathrm{~d} x(\Gamma) .
$$

We now choose, according to the previous property (2.12) $\delta_{1}$ such that meas $A<\delta_{1}=$ Sup $\int_{A}|f(g)|<\varepsilon / 8$, and $k>k_{0}$ such that meas $G_{k}<\delta_{1}$ where $G_{k}=\left\{x,\left|g_{k}\right|>R\right\} \cup$ $\int|G| \leq L$ $\{x,|g|>R\}$.

Choose also $k_{0}$ so that for $k>k_{0}$

We then write

$$
\mathrm{d} x \mid\left(\left|g(x)-g_{k}(x)\right| \geq \delta\right\}<\varepsilon / 8 \mathrm{~d} x(\Gamma) \max _{|\xi| \leq R}\|f(\xi)\| .
$$

$$
\begin{aligned}
\int\left|f\left(g_{k}\right)-f(g)\right| \leq & \int_{\substack{|g k-g|<\delta \\
\left|g_{k}\right|,|g| \leq 2 R}}\left|f\left(g_{k}\right)-f(g)\right|+\int_{\substack{\left|g_{k}-g\right|<\delta \\
\left|g_{k}\right|>2 R \text { or }|g|>2 R}}\left|f\left(g_{k}\right)-f(g)\right| \\
& +\int_{\substack{|g k-g|>\delta \\
\left|g_{k}\right|,|g| \leq 2 R}}\left|f\left(g_{k}\right)-f(g)\right| \\
\leq & \frac{\varepsilon}{4|\Gamma|}|\Gamma|+\frac{\varepsilon \operatorname{Sup}|f| \operatorname{mes} \Gamma}{4 \operatorname{Sup}|f| \operatorname{mes} \Gamma}+\int_{\substack{|g k-g|>\delta \\
\left|g_{k}\right|>2 R \text { or }|g|>2 R}}\left|f\left(g_{k}\right)-f(g)\right| .
\end{aligned}
$$

The last integral is arbitrarily small if we note that when $\delta$ is less than $R$, and $\left|g_{k}-g\right|<\delta$, $\left|g_{k}\right|>2 R$ implies $|g|>R$ and conversely. Then

$$
\begin{aligned}
& \int_{\substack{|g k-g|>2 R \text { or }|g|>2 R \\
\left|g_{k}-g\left(g_{k}\right)-f(g)\right|}}\left|\leq \int_{\{|g k|>R \text { and }|g|>R}\right| f\left(g_{k}\right)-f(g) \mid \\
& \leq \frac{\varepsilon}{4 L} \int\left|g_{k}\right|+\int|g| \\
& \leq \frac{\varepsilon}{2}
\end{aligned}
$$

and the proof is complete.

As in (1.25), theorem 2.4 has corollaries giving necessary and sufficient conditions for $f\left(\mu_{k}\right)-f(\mu)$ weak star in $C_{0}\left(\Omega, \mathbb{R}^{d}\right)^{\prime}$ or in $B C\left(\Omega, \mathbb{R}^{d}\right)^{\prime}$ with $\Omega$ open. The formulation and proof are left to the reader.

\section{3. $\Phi(\mu)$ WITH $\Phi$ ASYMPTOTICALLY HOMOGENEOUS}

Suppose $\Phi: \mathbb{R}^{d} \rightarrow \mathbb{R}$ has an asymptote in the sense that

$$
\lim _{t \rightarrow+\infty} \Phi(t \xi) /|t \xi| \equiv G(\xi)
$$

exists uniformly for $\xi$ in compact subsets of $\mathbb{R}^{d} \backslash 0$. Then $G$ is positive homogeneous of degree zero and if $F(\xi) \equiv|\xi| G(\xi), \Phi=F+f$ with $f$ sublinear. If $\Phi$ is continuous, then, so is $F$ and if $\Phi(0)=0$, then $f(0)=0$. This motivates the following definition. 
Definition. $\Phi \in C\left(\mathbb{R}^{d}, \mathbb{R}\right)$ is called asymptotically homogeneous iff

(i) $\Phi(0)=0$

(ii) $\lim _{t \rightarrow+\infty} \Phi(t \xi) / t$ exists uniformly on $|\xi|=1$.

The paragraph leading up to the definition establishes the following equivalence.

Proposition 3.1. $\Phi$ is asymptotically homogeneous if and only if $\Phi=F+f$ with $f, F \in C\left(\mathbb{R}^{d}, \mathbb{R}\right), F$ continuous and positive homogeneous of degree one and $f$ sublinear with $f(0)=0$. The functions $F$ and $f$ are uniquely determined by $\Phi$.

Definition. We suppose $\Gamma \subset \mathbb{R}^{n}$ is compact and $\mathrm{d} x$ is Lebesque measure on $\Gamma$. If $\Phi: \mathbb{R}^{d} \rightarrow \mathbb{R}$ is asymptotically homogeneous then $\Phi(\mu) \underset{\text { def }}{\bar{F}} F(\mu)+f(\mu)$ where $\Phi=F+f$ is the decomposition of proposition 3.1 and $F(\mu)$ and $f(\mu)$ are defined in Sections 1 and 2 respectively.

THEOREM 3.2. Suppose $\mu_{k}, \mu \in M\left(\Gamma, \mathbb{R}^{d}\right)$ have Lebesque decompositions $\mu_{k}=g_{k} \mathrm{~d} x+v_{k}$, $\mu=g \mathrm{~d} x+v$ and that $\mu_{k} \rightarrow \mu$. Then the following conditions are equivalent:

(I) $\Phi\left(\mu_{k}\right) \rightarrow \Phi(\mu)$ for all asymptotically homogeneous functions $\Phi$.

(II) $g_{k}$ converges to $g$ in measure and the equivalent conditions of theorem 1.15 hold.

Proof. The result follows immediately on combining theorem 1.15 and theorem 2.3.

In the case of scalar valued measures, that is $d=1$, the remark following example 1.10 shows that $\mu_{k}^{ \pm} \rightarrow \mu^{ \pm}$is sufficient to insure that $F\left(\mu_{k}\right) \rightarrow F(\mu)$. The next variation of that condition is useful in our study of semilinear hyperbolic systems [4].

Proposition 3.3. If $d=1$ and $\mu_{k}, \mu, g_{k}, g, v_{k}, v$ are as in theorem 3.2, then the following condition is equivalent to conditions I and II of theorem 3.2.

(III) $\left|\mu_{k}-g \mathrm{~d} x\right|-|v|$.

Proof. First we show that III implies that the conditions of theorem 2.3 hold. The lower semicontinuity of the variation yields

$$
|\mu| \leq \lim \inf \left|\mu_{k}\right| \leq \lim \inf \left|\mu_{k}-g \mathrm{~d} x\right|+|g| \mathrm{d} x=|\nu|+|g| \mathrm{d} x=|\mu| .
$$

It follows that $|\mu|=\lim \left|\mu_{k}\right|$ which verifies condition $\mathrm{B}$ of theorem 2.3 with the strictly convex $\operatorname{norm} \varphi(s)=|s|$.

To show that $g_{k}$ converges to $g$ in measure, observe that $\left|g_{k}-g\right| \mathrm{d} x \leq\left|\mu_{k}-g \mathrm{~d} x\right|$. Then for any $\eta>0$ fixed we must show that lim sup $\mathrm{d} x\left(A_{k}\right)=0$ where $A_{k} \equiv\left\{x \in \Gamma:\left|g_{k}(x)-g(x)\right| \geq \eta\right\}$. Choose a Lebesque null set, $M$, with $|v|(\Gamma \backslash M)=0$. For any $\varepsilon>0$ we will show that lim sup $\mathrm{d} x\left(A_{k}\right)<3 \varepsilon$. Given $\varepsilon$ choose compact $K_{1} \subset M$ with $|v|\left(M \backslash K_{1}\right)<\varepsilon \eta$. Choose compact $K_{2} \subset \Gamma \backslash M$ with $\mathrm{d} x\left(\left(\Gamma \backslash K_{2}\right)<\varepsilon\right.$. Choose $\varphi \in C(\Gamma)$ with $0 \leq \varphi \leq 1, \varphi=1$ on $K_{2}, \varphi=0$ on $K_{1}$. Then

$$
\eta \mathrm{d} x\left(A_{k} \cap K_{2}\right) \leq \int_{A_{k}}\left|g_{k}-g\right| \varphi \mathrm{d} x \leq \int\left|g_{k}-g\right| \varphi \mathrm{d} x .
$$

Using $\left|g_{k}-g\right| \mathrm{d} x \leq\left|\mu_{k}-g \mathrm{~d} x\right|$, it follows that

$$
\lim \sup \mathrm{d} x\left(A_{k} \cap K_{2}\right) \leq \eta^{-1} \lim \sup \left\langle\left|\mu_{k}-g \mathrm{~d} x\right|, \varphi\right\rangle=\eta^{-1}\langle|v|, \varphi\rangle<\varepsilon .
$$

However, $\mathrm{d} x\left(\Gamma \backslash K_{2}\right)<2 \varepsilon$, so lim sup $\mathrm{d} x\left(A_{k}\right)<3 \varepsilon$. 
This completes the proof that (III) implies the conditions of proposition 3.2. It remains to prove the converse.

We first remark that it suffices to prove the converse for nonnegative sequences $\mu_{k} \geq 0$. For arbitrary $\mu_{k}$ we apply the special case to the sequence $\mu_{k}^{+}-g^{+} \mathrm{d} x+v^{+}$which itself satisfies the conditions of proposition 3.2. This yields $\left|\mu_{k}^{+}-g^{+} \mathrm{d} x\right|-v^{+}$. Similarly $\left|\mu_{k}^{-}-g^{-} \mathrm{d} x\right| \rightarrow v^{-}$.

Since $\mu_{k}-g \mathrm{~d} x-\mu-g \mathrm{~d} x=\nu$, we have

$$
|v|=v^{+}+v^{-} \leq \liminf \left|\mu_{k}-g \mathrm{~d} x\right| \leq \lim \sup \left|\mu_{k}-g \mathrm{~d} x\right| .
$$

On the other hand, $\mu_{k}-g \mathrm{~d} x=\mu_{k}^{+}-g^{+} \mathrm{d} x+\mu_{k}^{-}-g^{-} \mathrm{d} x$, so

$$
\lim \sup \left|\mu_{k}-g \mathrm{~d} x\right| \leq \lim \sup \left\{\left|\mu_{k}^{+}-g^{+} \mathrm{d} x\right|+\left|\mu_{k}^{-}-g^{-} \mathrm{d} x\right|\right\}=v^{+}+v^{-}=|v| .
$$

It follows that $\left|\mu_{k}-g \mathrm{~d} x\right|-|v|$ which is the desired conclusion.

It remains to show that if $\mu_{k} \geq 0$ satisfy the hypotheses of proposition 3.2 then $\left|\mu_{k}-g \mathrm{~d} x\right| \rightarrow$ $|v|=v$. Toward that end, note that

$$
\left|\mu_{k}-g \mathrm{~d} x\right|=\mu_{k}-g \mathrm{~d} x+2\left(\mu_{k}-g \mathrm{~d} x\right)^{-}=\mu_{k}-g \mathrm{~d} x+2\left(g_{k}-g\right)^{-} \mathrm{d} x
$$

We next prove that $\left(g_{k}-g\right)^{-} \mathrm{d} x$ converges to zero in norm. Given $\varepsilon>0$, choose $\delta>0$ so that for any Borel set $A$ with $\mathrm{d} x(A)<\delta$, we have $\int_{A} g \mathrm{~d} x<\varepsilon / 2$. Choose $N$ so that for $n>N$,

$$
\mathrm{d} x\left\{x \in \Gamma:\left|g_{k}(x)-g(x)\right|>\varepsilon / 2 \mathrm{~d} x(\Gamma)\right\} \equiv \mathrm{d} x\left(A_{k}^{\varepsilon}\right)<\delta .
$$

Then,

$$
\int\left|\left(g_{k}-g\right)^{-}\right| \mathrm{d} x=\int_{0 \leq g_{k} \leq g}\left(g-g_{k}\right) \mathrm{d} x=\int_{A_{k}^{\xi_{k}} \cap\left\{g \geq g_{k}\right\}}+\int_{\left\{g \geq g_{k}\right\} \cap \Gamma \backslash \mathcal{A}_{k}^{\varepsilon}} .
$$

For $n>N$, the contribution of $A_{k}^{\varepsilon}$ is dominated by the integral of $g$ over $A_{k}^{\varepsilon}$, hence by $\varepsilon / 2$. The contribution of the second integral is also dominated by $\varepsilon / 2$. This proves the desired norm convergence.

It follows from (3.4) that $\left|\mu_{k}-g \mathrm{~d} x\right|$ and $\mu_{k}-g \mathrm{~d} x$ have the same weak limit so $\left|\mu_{k}-g \mathrm{~d} x\right| \rightarrow$ $v=|v|$. The proof of (III) for nonnegative $\mu_{k}$ satisfying the conditions of proposition 3.2 is complete.

$$
\text { 4. } f(x, \mu), F(x, \mu), \Phi(x, \mu)
$$

The results of the previous sections extend to functions which depend also on $x$. The modifications of the proofs are immediate. Here we describe an appropriate class of functions and the basic result.

Definition. $f \in C\left(\Gamma \times \mathbb{R}^{d}, \mathbb{R}\right)$ is sublinear if $f(x, 0)=0$ and $\lim _{\xi \rightarrow \infty} f(x, \xi) /|\xi|=0$ uniformly on $\Gamma$.

Definition. $F \in C\left(\Gamma \times \mathbb{R}^{d}, \mathbb{R}\right)$ is positively homogeneous of degree 1 iff $F(x, t \xi)=t F(x, \xi)$ for all $x, \xi \in \Gamma \times \mathbb{R}^{d}$ and $t>0$.

Definition. $\Phi \in C\left(\Gamma \times \mathbb{R}^{d}, \mathbb{R}\right)$ is asymptotically homogeneous iff $\Phi(x, 0)=0$ for all $x \in \Gamma$ and there is an $F \in C\left(\Gamma \times \mathbb{R}^{d}, \mathbb{R}\right)$ positively homogeneous of degree zero and a sublinear $f$ such that $\Phi=F+f$. 
It is no problem to modify the definitions of Sections 1,2 and 3 to define $F(x, \mu), f(x, \mu)$, $\Phi(x, \mu)$ the last two requiring $\Gamma \subset \mathbb{R}^{n}$ and depending explicitly on the normalized Lebesque measure $\mathrm{d} x$.

THEOREM 4.1. If $\mu_{k}, \mu$ satisfy the conditions of theorem 1.15 then $F\left(x, \mu_{k}\right)-F(x, \mu)$ for all positively homogeneous $F \in C\left(\Gamma \times \mathbb{R}^{d}: \mathbb{R}^{d}\right)$. If $\mu_{k}=g_{k} \mathrm{~d} x+v_{k}, \mu=g \mathrm{~d} x+v$ satisfy condition $(\gamma)$ of theorem 2.3 then $f\left(x, \mu_{k}\right) \rightarrow f(x, \mu)$ for all sublinear $f \in C\left(\Gamma \times \mathbb{R}^{d}: \mathbb{R}^{d}\right)$. If $\mu_{k}$ satisfies the conditions of theorem 1.15 and $\mu_{k} \rightarrow g$ in measure then $\Phi\left(x, \mu_{k}\right) \rightarrow \Phi(x, y)$ for all asymptotically homogeneous $\Phi$.

Proof. Left to reader.

\section{REFERENCES}

1. Bourbaki N., Integration, 2nd edition, Chapter 5. Hermann, Paris (1967).

2. Demengel F. \& Rauch J., Continuité faible des fonctions de mesures, C.r. Acad. Sci. Paris Série I, 63-65 (1987).

3. Demengel F. \& Rauch J., Superposition d'ondes singuliéres, C.r. Acad. Sci. Paris Série I, 15 (1987).

4. Demengel F. \& Rauch J., Measure valued solution of asymptotically homogeneous semilinear hyperbolic systems in dimension one, Proc. R. Soc. Edinb. (to appear).

5. Demengel F. \& Temam R., Convex function of a measure, Indiana Math. J. 33, 673-709 (1984).

6. DeMENGEL F. \& SERRE D., Non-vanishing singular parts for hyperbolic scalar equations with initial data measures (to be submitted).

7. FEDERER, Geometric Measure Theory. Springer, Berlin (1969).

8. GofFMAN \& SERRIN, Sublinear functions of measures and variational integrals, Duke math. J. 31, 159-178 (1964).

9. Rauch J. \& TaYlor B. A., The Dirichlet problem for the multidimensional Monge-Ampère equation, Rocky Mountain J. Math. 7, 345-364 (1977).

10. ReschetNYAK, Weak convergence of completely additive vector functions on a set. Sib. math. J. 9, 159-178 (1968).

11. Temam R., Problèmes Mathématiques en Plasticité, in Collection Méthodes Mathématiques de l'informatique. Gauthier-Villars, Paris (1983); English Translation, Gauthier-Villars, New York/(1985). 